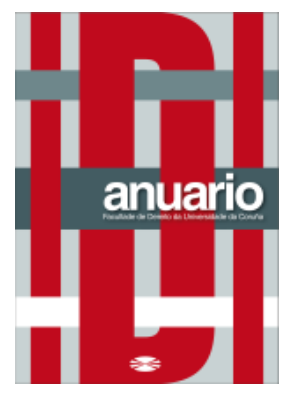

Anuario da Facultade de Dereito da Universidade da Coruña

Vol. 25 (2021), pp. 38-49

ISSNe: 2530-6324 || ISSN: 1138-039X

DOI: https://doi.org/10.17979/afdudc.2021.25.0.8797

\title{
LA VENTA DE LA UNIDAD PRODUCTIVA COMO SOLUCIÓN A LA INSOLVENCIA
}

\section{THE SALE OF THE PRODUCTION UNIT AS A SOLUTION TO INSOLVENCY}

\author{
CÉSAR GILO GÓMEZ \\ Abogado. Doctor en Derecho \\ Universidad de Salamanca \\ https://orcid.org/0000-0002-5492-6322
}

Recibido: $31 / 05 / 2021$

Aceptado: 07/10/2021

Resumen: En demasiadas ocasiones el patrimonio del deudor concursado es sometido a su liquidación para la satisfacción de sus acreedores. Aceptando que esta es la solución del concurso en la generalidad de los casos, la venta de todo o de una parte conjunta de los bienes del deudor en funcionamiento se erige en una opción que permite a través de un mero cambio de titularidad, la satisfacción de los acreedores y el mantenimiento de la actividad económica. Junto a estos objetivos, en la actualidad se explora la posibilidad de enajenar la unidad productiva con carácter previo a la declaración del concurso, para aprovechar las importantes ventajas que la anticipación del proceso de venta presenta, evitando así tanto la devaluación de los activos del deudor por el mero transcurso del tiempo como por la connotación negativa asociada a toda declaración del concurso.

Palabras clave: Unidad Productiva, Valoración, Trabajadores, Publicidad, Deudas, Venta.

Abstract: All too often, the assets of the insolvency debtor are subject to liquidation to the satisfaction of its creditors. Accepting that this is the solution of the contest on most cases, the sale of all or a joint part of the assets of the debtor in operation is an option that allows through a mere change of title, the satisfaction of the creditors and the maintenance of the economic activity. Along with these objectives, the possibility of disposing of the production unit prior to the announcement of the competition is currently being explored, in order to take advantage of the important advantages that the anticipation of the sale process 
presents, thus avoiding both the devaluation of the debtor's assets by the mere passage of time and the negative connotation associated with any declaration of the arrangement.

Keywords: Production Unit, Valuation, Employees, Advertising, Debts, Sale.

Sumario: I. INTRODUCCIÓN. II. DELIMITACIÓN DE LA UNIDAD PRODUCTIVA. III. LA VALORACIÓN DE LA UNIDAD PRODUCTIVA. IV. LA ASUNCIÓN DE LOS PASIVOS DE LA UNIDAD PRODUCTIVA. LIBERTAD DE PACTOS EN FUNCIÓN DEL PRECIO. V. LA IMPORTANCIA DE LA ENAJENACIÓN TEMPRANA. LA VENTA PRE-CONCURSAL. VI. LA PUBLICIDAD COMO MEDIO Y GARANTÍA PARA LA ENAJENACIÓN DE LA UNIDAD PRODUCTIVA. VII. LA DIRECTIVA (UE) 2019/1023, SOBRE MARCOS DE REESTRUCTURACIÓN PREVENTIVA Y SU REPERCUSIÓN EN LA VENTA DE UNIDADES PRODUCTIVAS. VIII. CONCLUSIONES. BIBLIOGRAFÍA.

$* \quad *$

\section{INTRODUCCIÓN}

La experiencia concursal en España a raíz de la modernización de las instituciones producida por la Ley 22/2003, de 9 de julio, Concursal, ha demostrado que la declaración judicial de insolvencia como solución de la misma ha sido un fracaso, obligando a acudir a otros mecanismos preconcursales o al amparo del propio concurso pero que permitan alcanzar una solución rápida y eficaz. En este sentido, la práctica ha enseñado que la apertura del procedimiento concursal, en lugar de solución, constituye parte del problema, ya sea por la demora de cualquier procedimiento judicial, la cual conlleva la consecuente depreciación de los activos del deudor durante su tramitación, como por la propia desconfianza que genera en la sociedad el mero hecho de acudir al mismo.

Tanto la actual regulación establecida en el Real Decreto Legislativo 1/2020, de 5 de mayo, por el que se aprueba el texto refundido de la Ley Concursal, como los últimos avances en España en materia de Pre-Pack dejan en evidencia que la venta de la unidad productiva del deudor es la mejor opción para solucionar su situación de insolvencia. Con ello se consiguen dos objetivos: mantener la actividad y conservar los puestos de trabajo, metas fundamentales para la supervivencia del tejido empresarial del país, que nos deben llevar a cumplir con el fin principal del procedimiento concursal: la satisfacción de los acreedores.

En este artículo se pretende reflexionar respecto a todo ello y aportar una visión panorámica de lo que parece constituirse por fin en la solución definitiva para las situaciones de insolvencia en nuestro país.

\section{DELIMITACIÓN DE LA UNIDAD PRODUCTIVA}


La propia normativa concursal recoge expresamente en su artículo 200 del Real Decreto Legislativo $1 / 2020$, de 5 de mayo, por el que se aprueba el texto refundido de la Ley Concursal - en adelante TRLC - el concepto de unidad productiva, definiendo el mismo como aquel conjunto de medios organizados para el ejercicio de una actividad económica esencial o accesoria, acogiendo de esta forma el legislador una delimitación propia de la regulación laboral ${ }^{1}$.

Teniendo en cuenta esta definición, deberá procederse a la valoración de referida unidad, partiendo de la premisa de que cualquier sistema organizado de bienes tiene un mayor valor que la consideración independiente de cada uno de los mismos ${ }^{2}$.

Por esta razón, la venta conjunta de todos o parte de los medios productivos del deudor en funcionamiento se erige como el mejor mecanismo para la maximización de sus activos y por ende, para la satisfacción de los acreedores con el producto de su venta.

El patrimonio del deudor puede estar compuesto por una o varias unidades productivas mientras que cada una de ellas mantenga la propiedad de poder ser considerada como independiente del resto. Corresponderá al deudor estudiar las ofertas existentes para determinar la entidad de la unidad productiva bajo el criterio principal de maximizar el beneficio obtenido, siendo interesante que la misma sea más o menos amplia y abarque más o menos elementos productivos en función del numerario de la oferta pero siempre sin romper la conjunción de medios expresamente prevista normativamente en el artículo 200 TRLC.

Si bien, en esta transmisión de medios no solo nos encontramos con elementos materiales, sino también con elementos humanos y obligaciones contractuales que deben determinarse en la venta y cuyos aspectos influirán notablemente en el precio final de adquisición.

\section{LA VALORACIÓN DE LA UNIDAD PRODUCTIVA}

El artículo 75.2 de la Ley 22/2003, de 9 de julio, Concursal —en adelante LC requería incluir en el informe de la Administración Concursal una valoración de la empresa en su conjunto y de las unidades productivas que la integrasen, un precepto sumamente importante ya que facilitaba información esencial para el concurso y para la transmisión de la unidad productiva, si bien es cierto que esta información se obtenía en un momento no inicial del procedimiento. A pesar de ello, otorgaba ya una valoración que permitía negociaciones que posibilitaran la venta de la unidad productiva, conociendo ya desde ese momento si era más interesante la venta conjunta o la venta de bienes y derechos de forma

\footnotetext{
${ }^{1}$ Artículo 44.2 del Real Decreto Legislativo 2/2015, de 23 de octubre, por el que se aprueba el texto refundido de la Ley del Estatuto de los Trabajadores.

${ }^{2}$ Respecto a ello, el profesor RUBIO VICENTE destaca que con la incorporación de esta definición al TRLC se resuelven las dudas interpretativas surgidas de su utilización en diversos preceptos de la anterior Ley Concursal como el artículo 146 bis. Vid. RUBIO VICENTE, P.J, «Las especialidades de la enajenación de unidades productivas en el nuevo Texto Refundido de la Ley Concursal», en Revista de Derecho Concursal y Paraconcursal, $\mathrm{n}^{\circ}$ 33, Sección Ponencias y Estudios, Segundo semestre de 2020, Wolters Kluwer, págs. 49-78.
} 
individual. Mencionado precepto no ha sido trasladado al TRLC, ya que el artículo 293.2 TRLC no hace referencia a la valoración de la unidad productiva del deudor, sino sólo de aquellas empresas que formaran parte de la masa activa del deudor ${ }^{3}$.

Con independencia de lo anterior, en el supuesto de que la venta de la unidad productiva se produzca antes de la declaración del propio procedimiento concursal, su valoración deberá necesariamente producirse extramuros del concurso, por lo que el informe de la Administración Concursal nada podrá aportar en este sentido.

En esta situación, y ante la ausencia de pronunciamiento por parte de la Administración Concursal, cobra especial importancia acudir a entidades especializadas que por su objeto social conocen el mercado y pueden tanto valorar la unidad productiva, como poner en contacto a oferentes y vendedores, de cara a la futura venta de la misma ${ }^{4}$.

Debe tenerse en cuenta que el artículo 216 TRLC prevé la posibilidad de que el Juez autorice en cualquier estado del concurso la enajenación a través de este tipo de entidades, para lo que es necesario la previa valoración de la unidad productiva por parte de las mismas.

La regulación de las entidades especializadas aparece en un texto extraconcursal, ya que su definición se contiene en el artículo 641 de la Ley 1/2000, de 7 de enero, de Enjuiciamiento Civil, en el que se prevén como personas o entidades conocedoras del mercado en que se compran y venden determinados bienes y que poseen los requisitos legalmente exigidos para operar en ese concreto mercado. Sin embargo, su regulación puede calificarse como incompleta, al dedicarse un único artículo a las mismas -a diferencia de los preceptos exclusivamente destinados a regular la subasta- además de provocar una serie de gastos que hace que su aplicación sea mucho más residual que la subasta en la generalidad de las ejecuciones.

Sin embargo, en el ámbito concursal, donde la valoración de la unidad productiva puede ser necesaria sin tener que acudir a un procedimiento de subasta, sus inconvenientes disminuyen, aumentando sus ventajas. En este sentido, el hecho de que la tasación de la unidad productiva en funcionamiento se efectúe por expertos acercará mucho las posturas entre deudor concursado o cercano a la insolvencia y potenciales compradores.

\footnotetext{
${ }^{3}$ No comparte este extremo RIPOL CARULLA, quien entiende que si bien la redacción del apartado segundo del artículo 293 TRLC resulta confusa y pudiera conducir a esa conclusión, no existen motivos que justifiquen el cambio de contenido del artículo. Vid. RIPOL CARULLA, I., «Valoración de empresas en el marco de la transmisión de unidades productivas en el Texto Refundido de la Ley Concursal», en Revista de Derecho Concursal y Paraconcursal, $\mathrm{n}^{\mathrm{0}} 34$, Sección Cuestiones prácticas, Primer semestre de 2021, Wolters Kluwer, págs. 269-280.

${ }^{4}$ Vid. respecto a las mismas ACHÓN BRUÑÉN, M.J., «La venta por persona o entidad especializada como mecanismo de enajenación forzosa: reformas legales que serían aconsejables para potenciar su aplicación en la práctica», en Práctica de Tribunales, n ${ }^{\circ}$ 113, Sección Tribuna Libre, marzo-abril 2015, Wolters Kluwer, quien destaca que su regulación es parca y llena de lagunas, lo que provoca que las mismas se miren con recelo.
} 
De igual forma, la existencia de entidades especializadas que actúan de forma telemática ha permitido reducir los costes, tanto de la transacción como de la propia publicidad, adquiriendo en la actualidad una importancia sobresaliente el portal de subastas del Consejo General de Procuradores de España tras las recomendaciones efectuadas desde distintos Juzgados de lo Mercantil ${ }^{5}$.

\section{LA ASUNCIÓN DE LOS PASIVOS DE LA UNIDAD PRODUCTIVA. LIBERTAD DE PACTOS EN FUNCIÓN DEL PRECIO}

El artículo 224 TRLC faculta la libertad de pactos en la negociación que se lleve a cabo para la adquisición de la unidad productiva, previendo expresamente la posibilidad de que el adquirente no asuma la obligación de pago de los créditos no satisfechos por el concursado antes de la transmisión, salvo excepciones ${ }^{6}$.

De este modo, el legislador ha querido otorgar flexibilidad a este mecanismo, facilitando en la medida de lo posible la adaptación a todas las circunstancias que puedan producirse en una negociación de venta de estas características, para tratar así de trabar la misma lo menos posible, ya que no olvidemos que en muchas ocasiones, el primordial atractivo para el comprador es adquirir una empresa ya en funcionamiento sin asumir las cargas de la misma.

La principal excepción al régimen de "libertad de transmisión sin cargas" la constituye una materia en la que entronca el régimen mercantil con el laboral, esto es, en aquellos supuestos en los que la transmisión de la unidad productiva constituya una sucesión de empresa al amparo del artículo 44 del Real Decreto Legislativo 2/2015, de 23 de octubre, por el que se aprueba el texto refundido de la Ley del Estatuto de los Trabajadores. Y es que no debe olvidarse que el comprador no ostenta ningún tipo de legitimación ni interés en el procedimiento concursal, por lo que no puede serle de aplicación la normativa concursal, sino que el mismo se verá sometido por la regulación laboral.

Sin embargo, se ha querido facilitar de la mejor manera posible a los adquirentes de unidades productivas para que no tengan que cargar con créditos laborales y de Seguridad Social, en aras de mantener el empleo. Un difícil equilibrio que actualmente se salda en

${ }^{5} \mathrm{Vid}$. las críticas que se han producido en este sentido en MARTÍ MIRAVALLS, J., «Liquidación concursal y subasta telemática por entidad especializada», en Revista de Derecho Concursal y Paraconcursal, $\mathrm{n}^{\circ}$ 34, Sección Varia, Primer semestre de 2021, Wolters Kluwer, págs. 139-154, quien entiende que todas las entidades especializadas, no sólo el Consejo General de Procuradores, garantizan un proceso público, transparente y concurrente, no compartiendo la preferencia actual de los Tribunales por la plataforma propia de la Procura en detrimento del resto.

${ }^{6}$ De las tres excepciones recogidas en el precepto, las dos primeras son fácilmente previsibles, ya que se refieren por un lado a la adquisición expresa del compromiso de pago de las deudas por parte del adquirente o cuando así se establezca normativamente. Es la tercera de ellas relativa a los supuestos de sucesión de empresa en los que se obliga al adquirente respecto de los créditos laborales y de seguridad social de los trabajadores en cuyos contratos quede subrogado el adquirente. 
beneficio del adquirente, quien se hace cargo únicamente de los contratos de los trabajadores que se mantienen en la unidad productiva transmitida ${ }^{7}$.

Además de todo ello, deben ser valoradas las consecuencias de la enajenación en cuanto a los contratos que pudieran haber sido suscritos con anterioridad a la transmisión de la unidad productiva y que estuvieran afectos a la misma. En este sentido, el artículo 222 TRLC establece la subrogación automática del adquirente en los mismos, así como en las licencias que formen parte de la unidad productiva ${ }^{8}$. Ahora bien, la excepción la constituyen los contratos de carácter administrativo que estuvieran vigentes, respecto a los cuales no se produce una subrogación automática, sino una remisión a la legislación sobre los contratos públicos (particularmente al Real Decreto Legislativo 3/2011, de 14 de noviembre, por el que se aprueba el texto refundido de la Ley de Contratos del Sector Público) $)^{9}$.

Particularmente relevante se plantea la responsabilidad del cesionario respecto a las deudas que la cedente tenga con la Administración y que pudiera constituir algún tipo de impedimento para la continuación de los contratos administrativos suscritos y que pudieran ser fundamentales para la continuidad del negocio y por ende, aspecto clave en la negociación para la compraventa.

\section{LA IMPORTANCIA DE LA ENAJENACIÓN TEMPRANA. LA VENTA PRE- CONCURSAL}

Nada se indica en nuestra normativa concursal respecto a la enajenación de la unidad productiva antes de iniciarse el procedimiento concursal, girando todas las previsiones normativas en enajenaciones, como muy tempranas, en la fase común, de acuerdo con la interpretación que puede efectuarse del artículo 205 TRLC.

No obstante, el artículo 215 TRLC prevé expresamente la posibilidad de que se produzca la enajenación de las unidades productivas en cualquier estado del concurso,

\footnotetext{
${ }^{7}$ Respecto a esta cuestión, vid. la reflexión que se efectúa en LÓPEZ CUMBRE, L., «Nada es lo que parece. El régimen concursal laboral de la venta de unidad productiva no cambia», en Diario La Ley, $\mathrm{n}^{\circ}$ 9843, Sección Tribuna, 5 de mayo de 2021, Wolters Kluwer, quien avisa del peligro de fraude de un sistema que incentiva la persecución de un beneficio fácil mediante la adquisición sin cargas de la unidad productiva para volver a aparecer con otra marca en el mercado.

${ }^{8}$ Mencionado precepto no prevé la necesidad de que se produzca consentimiento de la contraparte en los contratos objeto de subrogación. No obstante, parte de la Doctrina, aboga por la aprobación de un protocolo donde se recomiende comunicar a las contrapartes de los contratos vigentes afectos a la viabilidad del proyecto empresarial, la existencia y el resultado del proceso de transmisión de la unidad productiva. Así, vid. VERDUGO GARCÍA, J; FARIÑAS PEÑA, J.M «Transmisiones de unidades productivas y cesiones de contratos: ¿deben comunicarse a las contrapartes cedidas?», en Revista de Derecho Concursal y araconcursal, $n^{0}$ 34, Sección Cuestiones prácticas, Primer semestre de 2021, Wolters Kluwer, págs. 259-268.

${ }^{9}$ Vid. en este sentido FARIÑAS PENA, J.M, «Cesión de contratos administrativos en la venta de unidades productivas en sede concursal», en Revista de Derecho Concursal y Paraconcursal, n 33, Sección Cuestiones prácticas, Segundo semestre de 2020, Wolters Kluwer, págs. 477-490, quien apuesta porque el Juez del concurso sea quien tenga el papel principal en esta materia para que no se deje la cesión de los contratos administrativos en manos del órgano de contratación ante el peligro de que la Administración rechace autorizar la cesión del contrato.
} 
previsión que otorga el necesario respaldo legal para poder considerar la venta de la unidad productiva antes incluso de que el procedimiento concursal sea declarado.

Ello viene refrendado de alguna manera por los artículos 523 y 530 TRLC, los cuales contemplan que el deudor presente junto con la solicitud de concurso, un plan de liquidación con una propuesta de adquisición de la unidad productiva, previsión normativa que daría amparo a que la negociación de la venta se produjera en un momento previo a la declaración del procedimiento concursal, autorizándose con en su propia declaración.

La principal ventaja de la venta de la unidad productiva en un momento previo a la declaración de concurso viene constituida por la necesidad de evitar la depreciación de los activos del deudor fruto de la lógica demora propia de toda tramitación procesal y de la habitual imagen negativa asociada a una situación de insolvencia. Por esta razón, si se vende la unidad productiva del deudor antes de declararse el concurso, se evita tanto la depreciación de activos como la devaluación de la imagen de la unidad productiva objeto de venta, maximizando de esta forma su valor y por tanto el grado de satisfacción de los acreedores.

Además de ello, esta modalidad se presenta como una opción más que interesante para paliar los problemas de liquidez por los que puede atravesar el concursado, situación en la que una venta rápida de la unidad productiva puede ser la solución que evite la situación de insolvencia.

La venta de la unidad productiva con carácter previo al inicio del procedimiento concursal ha pasado a ocupar la principal novedad del derecho concursal español desde que el pasado mes de enero de 2021 los Jueces de lo Mercantil de Barcelona en su Seminario de 20 de enero de 2021 establecieron una serie de Directrices para la aplicación en España del denominado sistema Pre-Pack ${ }^{10}$.

Nos encontramos ante un mecanismo a través del cual el deudor en situación de insolvencia actual o inminente puede en una fase preconcursal comunicar al Juzgado al que le correspondería conocer de su concurso, que va a iniciar negociaciones para la transmisión del negocio — utilizando para ello la vía del artículo 583 TRLC - solicitando el nombramiento de un experto independiente que supervise la operación. Este experto independiente se encargará de garantizar que se cumple con la necesaria publicidad y transparencia en la venta, el cual además intervendrá en la propia negociación con los acreedores, desde una posición completamente independiente e imparcial.

El proceso finaliza con la declaración del concurso, instante en el que se da traslado del Auto de declaración de concurso a los interesados para que se pronuncien respecto a la venta que se va a llevar a cabo. A continuación, el Juez del concurso, previo informe

10 Es lo cierto que mencionado mecanismo ya venía siendo aplicado con anterioridad en otros ordenamientos como Reino Unido, Holanda o EEUU, pero no ha sido hasta el Seminario de Jueces de lo Mercantil de Barcelona cuando se ha comenzado a utilizar en España. 
motivado del experto independiente — posterior Administración Concursal— adjudica la unidad productiva al oferente vía artículo 530 TRLC.

Con posterioridad a los Jueces de lo Mercantil de Barcelona, los Jueces de lo Mercantil de Madrid también han publicado su posición en este sentido ${ }^{11}$. La principal diferencia entre ambos protocolos reside en que las normas aprobadas desde Barcelona permiten la venta de la unidad productiva antes de declararse el concurso, a diferencia de las directrices de los Juzgados madrileños, que apuestan porque la venta se produzca en el seno del procedimiento concursal.

Finalmente, los Juzgados de Palma de Mallorca también han aprobado su Protocolo, que si bien con ciertos matices como la ausencia de remisión a organismo público alguno a efectos de informar y publicitar los datos de la unidad productiva, tiene importantes semejanzas con el aprobado por los Jueces barceloneses ${ }^{12}$.

\section{LA PUBLICIDAD COMO MEDIO Y GARANTÍA PARA LA ENAJENACIÓN DE LA UNIDAD PRODUCTIVA}

La publicidad de los distintos acontecimientos que se suceden en todo procedimiento concursal es esencial para conseguir el fin del propio procedimiento, garantizando los derechos de todos los interesados.

En la venta de la unidad productiva, esta garantía adquiere una especial relevancia, ya que cuanto mayor sea la repercusión de la noticia, más cantidad de ofertas de adquisición de la misma se presentarán y por ende, es de esperar que se produzca la maximización del precio de venta. A mayor abundamiento, esta misma publicidad conllevará que todos aquellos interesados vean suficientemente tutelados sus derechos con la consecuente audiencia para alegar lo que estimen oportuno respecto a la enajenación de la unidad productiva ${ }^{13}$.

Consciente de ello, la normativa concursal prevé en el artículo 423 TRLC la remisión al Registro Público Concursal de la información necesaria para facilitar la venta de la masa activa de aquellas personas jurídicas en liquidación. Entendemos que la aplicación de este precepto no debe reservarse exclusivamente a situaciones de empresas en liquidación, sino que debe utilizarse en la generalidad de los casos para facilitar la presentación de ofertas en este sentido y su posible valoración.

${ }^{11}$ Referidas medidas han sido plasmadas en un documento denominado Guía de Buenas Prácticas Procesales en materia del artículo 530 TRLC, aprobado por los Jueces Mercantiles de Madrid en reunión de 22 de enero de 2021, inserta en la Guía de buenas prácticas para la venta de unidades productivas publicada por el Ilustre Colegio de Abogados de Madrid.

${ }^{12} \mathrm{Vid}$. al respecto el Protocolo aprobado por la Junta Sectorial de Jueces de lo Mercantil de Baleares de fecha 28 de abril de 2021.

${ }^{13}$ Para facilitar la venta, la Administración Concursal debe hacer llegar la información al mayor número de interesados, pudiendo incluso invitar expresamente. Vid. en este sentido ARGÜELLES MORA, A, «La venta de unidades productivas en sede concursal como vía para la salvaguarda del tejido empresarial en la nueva Ley Concursal. RDL 1/2020», en Diario La Ley, No 9826, Sección Tribuna, 9 de abril de 2021, Wolters Kluwer, quien destaca la publicidad como uno de los factores clave en la venta de la unidad productiva. 
Por esta razón, concebimos razonable que se haga uso también de la publicidad complementaria prevista legalmente aplicada a la venta de la unidad productiva, para tratar así de llegar al mayor número de interesados posibles, maximizando de esta forma el precio de adquisición de la misma ${ }^{14}$.

Cuestión distinta es que se desee ofertar la venta de la unidad productiva en fase preconcursal mediante Pre-Pack. Debe tenerse en cuenta que en ese caso nos encontramos en un estadio previo a la declaración de concurso, por lo que no podrá utilizarse el régimen de publicidad previsto para el mismo. Es en esta fase donde entendemos que mayor relevancia adquieren las entidades especializadas, ya que ellas, además de valorar la unidad productiva, podrán poner en contacto al deudor con potenciales adquirentes con quien, de no acudir a las mismas, sería complicado contactar ${ }^{15}$.

\section{LA DIRECTIVA (UE) 2019/1023, SOBRE MARCOS DE REESTRUCTURACIÓN PREVENTIVA Y SU REPERCUSIÓN EN LA VENTA DE UNIDADES PRODUCTIVAS}

La trasposición al ordenamiento español de la Directiva (UE) 2019/1023, sobre marcos de reestructuración preventiva, medidas para aumentar la eficiencia de los procedimientos de reestructuración, insolvencia y exoneración de deuda, prevista para el próximo 17 de julio de 2021, se espera que sirva para terminar con la ausencia de regulación normativa expresa en España de determinadas instituciones preconcursales y que actualmente es colmada por parte de la Jurisprudencia ${ }^{16}$.

La razón la encontramos en que la Directiva prevé el fomento de soluciones preconcursales que casan perfectamente con los mecanismos de Pre-Pack que se pretenden implantar en España y que se espera otorgue a nuestra normativa los instrumentos necesarios para culminar con éxito la posibilidad de enajenar unidades productivas con antelación a la apertura del procedimiento concursal.

No obstante lo expuesto, la normativa Europea no se olvida de las enajenaciones de unidades productivas en la fase puramente concursal. En este sentido, el Considerando 4 de la misma, deja claro que lo que se pretende es favorecer planteamientos que tengan por objeto la recuperación de las empresas o al menos el rescate de aquellas de sus unidades

${ }^{14}$ La magistrada CÓRDOBA ARDAO ha venido insistiendo en una mayor implicación de las Administración públicas en los procesos de venta de las unidades productivas ya que el objetivo de mantenimiento del tejido industrial y conservación de puestos de trabajo redunda en la economía en general. Vid. CÓRDOBA ARDAO, B, «Los retos futuros de las ventas de unidades productivas en sede concursal», en Diario La Ley, No 9617, 21 de abril de 2020, Wolters Kluwer.

${ }^{15}$ No debe olvidarse que, conforme hemos señalado, la venta de la unidad productiva en fase preconcursal busca cierta discreción para evitar que los activos del deudor se devalúen por la proximidad de la insolvencia y la imagen negativa asociada a la misma. Por este motivo, la publicidad que pueda darse a esta situación debe venir de la mano de la necesaria prudencia.

${ }^{16}$ El magistrado PEDROSA DEL PINO advierte que de la forma en la que se trasponga la Directiva en nuestro país y del comportamiento de cada empresa en esta época pandémica, va a depender en gran medida la supervivencia del tejido empresarial español. Vid. PEDROSA DEL PINO. J, «Cash is king», en Diario La Ley, $\mathrm{n}^{\circ}$ 9846, Sección Tribuna, 10 de mayo de 2021, Wolters Kluwer. 
que sigan siendo económicamente viables, por lo que se espera que con la trasposición de la Directiva europea, se amplíe nuestra normativa tanto en fase preconcursal como en el propio seno del concurso ${ }^{17}$.

Así, sistemas expresamente previstos en la Directiva (UE) 2019/1023 como las alertas tempranas tienen como objetivo evitar tener que acudir a la liquidación concursal para conseguir la continuación de la actividad empresarial, lo que en ningún caso contradice la posibilidad de iniciar negociaciones cuanto antes para realizar la venta conjunta de la unidad productiva que favorezca a todas las partes implicadas ${ }^{18}$.

\section{CONCLUSIONES}

La venta de la unidad productiva se erige como una de las principales soluciones para enmendar la situación de insolvencia que obliga a acudir al procedimiento concursal. $\mathrm{Y}$ es que actualmente, como corolario de la apertura del concurso de acreedores y su propia tramitación es el irremediable fin en liquidación de los activos del deudor.

Por este motivo, la venta de la unidad productiva representa en la mayoría de las ocasiones el modo de solucionar la insolvencia y maximizar el producto de la venta de los activos.

Unido a ello, los últimos meses han visto aflorar en España un mecanismo de venta de la unidad productiva que pretende minimizar al máximo la posible depreciación de los bienes del deudor a causa de la declaración de concurso mediante su venta con anterioridad a que el propio concurso se declare (Pre-pack). Este modo de enajenación constituye una solución ideal en la mayoría de los casos, aunque sigue encontrando como dificultad el hecho de localizar a un tercer adquirente dispuesto a ofrecer el importe en el que se valore la unidad productiva.

Por esta razón, mecanismos como la publicidad complementaria que pueda otorgarse a la venta o confiar la misma a entidades especializadas, se convierten en fundamentales para que el procedimiento culmine con la necesaria rapidez y eficacia. Todo ello acompañado de las modificaciones legislativas que se espera se produzcan a raíz de la trasposición de la Directiva (UE) 2019/1023 a partir del 17 de julio de 2021.

17 Vid. en este sentido COHEN BENCHETRIT A, «La venta de la unidad productiva en el nuevo escenario concursal», en Revista de Derecho Concursal y Paraconcursal, no 33, Sección Ponencias, Segundo semestre de 2020, Wolters Kluwer, págs. 79-90, quien señala que, a pesar de que la Directiva pone acento en los mecanismos preconcursales, no deja de lado las enajenaciones de unidades productivas en fase puramente concursal, debiendo adquirir relevancia en el futuro este tipo de enajenaciones como vía para recuperar el tejido empresarial y los puestos de trabajo.

${ }^{18}$ Vid. LLORET VILLOTA, J.; CAICOYA CECCHINI, J,; LORENTE SIBINA, R., «"ALERTAS TEMPRANAS”. La aplicación a la situación en España», en Diario La Ley, n 9640, Sección Tribuna, 26 de mayo de 2020, Wolters Kluwer, quienes entienden que el concurso ha perdido su atractivo al reducir el legislador el abanico de empresas con posibilidad de continuar bajo el esquema de transmisión de unidad productiva, motivo por la que desde la Unión Europea se apuesta en la Directiva UE 2019/1023 por centrar los esfuerzos en la reestructuración preventiva, voluntaria y temprana. 


\section{BIBLIOGRAFÍA}

ACHÓN BRUÑÉN, M.J., «La venta por persona o entidad especializada como mecanismo de enajenación forzosa: reformas legales que serían aconsejables para potenciar su aplicación en la práctica», en Práctica de Tribunales, $\mathrm{n}^{\circ}$ 113, Sección Tribuna Libre, marzo-abril 2015, Wolters Kluwer.

ARGÜELLES MORA, A, «La venta de unidades productivas en sede concursal como vía para la salvaguarda del tejido empresarial en la nueva Ley Concursal. RDL 1/2020», en Diario La Ley, № 9826, Sección Tribuna, 9 de abril de 2021, Wolters Kluwer.

COHEN BENCHETRIT A, «La venta de la unidad productiva en el nuevo escenario concursal», en Revista de Derecho Concursal y Paraconcursal, $n^{\circ}$ 33, Sección Ponencias, Segundo semestre de 2020, Wolters Kluwer, págs. 79-90.

CÓRDOBA ARDAO, B, «Los retos futuros de las ventas de unidades productivas en sede concursal», en Diario La Ley, No 9617, 21 de abril de 2020, Wolters Kluwer.

FARIÑAS PENA, J.M, «Cesión de contratos administrativos en la venta de unidades productivas en sede concursal», en Revista de Derecho Concursal y Paraconcursal, $\mathrm{n}^{\mathbf{0}} 33$, Sección Cuestiones prácticas, Segundo semestre de 2020, Wolters Kluwer, págs. 477-490.

LLORET VILLOTA, J.; CAICOYA CECCHINI, J.; LORENTE SIBINA, R., «“ALERTAS TEMPRANAS". La aplicación a la situación en España», en Diario La Ley, n 9640, Sección Tribuna, 26 de mayo de 2020, Wolters Kluwer

LÓPEZ CUMBRE, L., «Nada es lo que parece. El régimen concursal laboral de la venta de unidad productiva no cambia», en Diario La Ley, n 9843, Sección Tribuna, 5 de mayo de 2021, Wolters Kluwer.

MARTÍ MIRAVALLS, J., «Liquidación concursal y subasta telemática por entidad especializada», en Revista de Derecho Concursal y Paraconcursal, n 34 , Sección Varia, Primer semestre de 2021, Wolters Kluwer, págs. 139-154.

PEDROSA DEL PINO. J., «Cash is king», en Diario La Ley, n 9846, Sección Tribuna, 10 de mayo de 2021, Wolters Kluwer.

RIPOL CARULLA, I., «Valoración de empresas en el marco de la transmisión de unidades productivas en el Texto Refundido de la Ley Concursal», en Revista de Derecho Concursal y Paraconcursal, $\mathrm{n}^{\mathrm{o}}$ 34, Sección Cuestiones prácticas, Primer semestre de 2021, Wolters Kluwer, págs. 269-280.

RUBIO VICENTE, P.J., «Las especialidades de la enajenación de unidades productivas en el nuevo Texto Refundido de la Ley Concursal», en Revista de Derecho Concursal y Paraconcursal, $\mathrm{n}^{\mathrm{o}}$ 33, Sección Ponencias y Estudios, Segundo semestre de 2020, Wolters Kluwer, págs. 49-78. 
VERDUGO GARCÍA, J.; FARIÑAS PEÑA, J.M., «Transmisiones de unidades productivas y cesiones de contratos: ¿deben comunicarse a las contrapartes cedidas?», en Revista de Derecho Concursal y Paraconcursal, $\mathrm{n}^{0}$ 34, Sección Cuestiones prácticas, Primer semestre de 2021, Wolters Kluwer, págs. 259-268. 